\title{
Correction to: Cerebral Microvascular Injury in Patients with Left Ventricular Assist Device: a Neuropathological Study
}

\author{
Tracey H. Fan ${ }^{1}$ - Sung-Min Cho ${ }^{2}\left(\mathbb{D} \cdot\right.$ Richard A. Prayson $^{3} \cdot$ Catherine E. Hassett $^{4} \cdot$ Randall C. Starling $^{5} \cdot$ Ken Uchino $^{4}(\mathbb{0}$
}

Published online: 19 October 2021

○) Springer Science+Business Media, LLC, part of Springer Nature 2021

\section{Correction to: Translational Stroke Research https://doi.org/10.1007/s12975-021-00935-z}

In Table 3, which all the numbers listed with percentage, the percentages should be inside a parenthesis as shown below:

The original article has been corrected.

Publisher's Note Springer Nature remains neutral with regard to jurisdictional claims in published maps and institutional affiliations.

The original article can be found online at https://doi.org/10.1007/ s12975-021-00935-z.

Ken Uchino

uchinok@ccf.org

1 Department of Neurology, Neurological Institute, Cleveland Clinic, Cleveland, OH, USA

2 Neurocritical Care Division, Departments of Neurology, Neurosurgery, Anesthesiology and Critical Care Medicine, Johns Hopkins Hospital, Baltimore, MD, USA

3 Department of Anatomic Pathology, Pathology \& Laboratory Medicine Institute, Cleveland Clinic, Cleveland, OH, USA

4 Cerebrovascular Center, Neurological Institute, Cleveland Clinic, 9500 Euclid Avenue/S80, Cleveland, OH, USA

5 Department of Cardiovascular Medicine, Heart, Vascular and Thoracic Institute, Kaufman Center for Heart Failure, Cleveland Clinic, 9500 Euclid Avenue/S80, Cleveland, OH, USA 
Table 3 Comparison of Patients with and without Cerebral Microbleeds

\begin{tabular}{llll}
\hline Patient characteristics & $\begin{array}{l}\text { Patient with CMBs } \\
\mathrm{n}=17\end{array}$ & $\begin{array}{l}\text { Patient without CMBs } \\
\mathrm{n}=4\end{array}$ & P value \\
\hline Age, median (IQR) & $48(31-63)$ & $64(58-69)$ & 0.12 \\
Male, $\mathrm{n}(\%)$ & $13(76 \%)$ & $1(25 \%)$ & 0.28 \\
Body mass index, median (IQR) & $29(26-33)$ & $24(23-28)$ & 0.34 \\
Past Medical History, n (\%) & & & \\
Diabetes mellitus & $7(41 \%)$ & $1(25 \%)$ & 1 \\
Hypertension & $9(53 \%)$ & $2(50 \%)$ & 1 \\
Arrhythmia & $11(65 \%)$ & $2(50 \%)$ & 0.6 \\
Chronic kidney disease & $5(29 \%)$ & $1(25 \%)$ & 1 \\
Pre-LVAD ischemic stroke & 0 & $1(25 \%)$ & 0.22 \\
Pre-LVAD hemorrhagic stroke & 0 & 0 & $\mathrm{NA}$ \\
LVAD support time, days, median (IQR) & $46(7-239)$ & $612(337-853)$ & 0.02 \\
LVAD brand- Heartmate II, n (\%) & $11(65 \%)$ & $3(75 \%)$ & 1 \\
Complications during LVAD course, n $(\%)$ & & & \\
Bloodstream or LVAD Infections & $9(53 \%)$ & $3(75 \%)$ & 0.59 \\
Cerebral infarct & $5(29 \%)$ & $3(75 \%)$ & 0.25 \\
Intraparenchymal hemorrhage & $3(17 \%)$ & $3(75 \%)$ & 0.06 \\
Pump thrombosis & $1(6 \%)$ & $1(25 \%)$ & 0.35 \\
Deep vein thrombosis & $3(17 \%)$ & 0 & 1 \\
Pulmonary embolism & 0 & $1(25 \%)$ & 1 \\
Significant non-intracranial bleeding & $7(41 \%)$ & $2(50 \%)$ & 0.58 \\
Disseminated intravascular coagulation & $1(6 \%)$ & 0 & 1 \\
\hline
\end{tabular}

IQR- interquartile range; LVAD, left ventricular assist device 